\title{
96. Relation entre la taille des neurones et leur époque d'apparition dans la moelle épinière chez le poulet
}

\section{Etude autoradiographique et caryométrique}

\author{
Par Akira KANEMitsU*) \\ (Comm. by Teizo Ogawa, M. J. A., April 12, 1971)
}

Au moyen de l'autoradiographie à l'aide de la thymidine tritiée on peut observer l'époque d'apparition de neurones d'une façon exacte. ${ }^{1)} \quad$ Récemment plusieurs auteurs ${ }^{2)}$ ont indiqué que les macroneurones se différencient plus tôt que les microneurones, mais il ne nous semble pas qu'il y ait eu de recherches systématiques sur ce sujet. Dans notre note autoradiographique précédente ${ }^{3)}$ nous avons examiné la distribution des éléments nerveux, différenciés à des stades différents dans la moelle épinière chez le poulet et signalé en bref que les élèments sont d'autant plus grands qu'ils apparaissent plus tôt. Cette fois, nous avons essayé d'analyser statistiquement la relation entre la taille des noyaux cellulaires et leur époque d'origine.

Ces recherches ont été éffectuées sur l'enflement cervical des embryons de poulet de race Leghorn blanche. Nous avons examiné 6 embryons fixés au stade 36, qui ont reçu au préalable une injection unique de $20 \mu \mathrm{Ci}$ de thymidine tritiée (activité spécifique $=5.0 \mathrm{Ci}$ / $\mathrm{mM}$ ) dans le sac du jaune d'oeuf au stade 17 (A), 20 (B), 23 (C), 26 (D), 29 (E) et 30 (F) respectivement (Fig. 1). Les stades de développement se réfèrent à la série établie par Hamburger et Hamilton (1951).4) Les préparations ont été fixées à la solution de Bodian II et inclues dans de la paraffine. Nous avons préparé

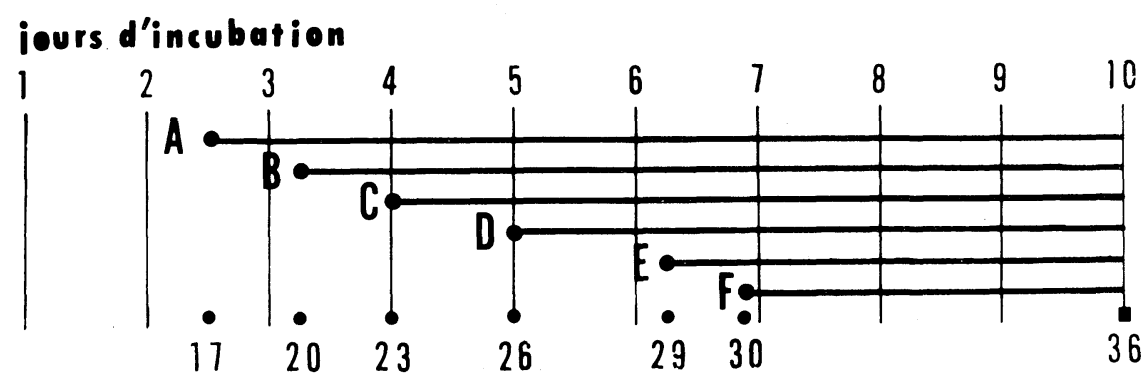

Fig. 1. Plan des expériences. Le point (•) et le carré (ם) représentent respectivement les stades d'injection et de fixation.

*) Institut de la Recherche du Cerveau, Faculté de Médecine, Université de Tokyo. 


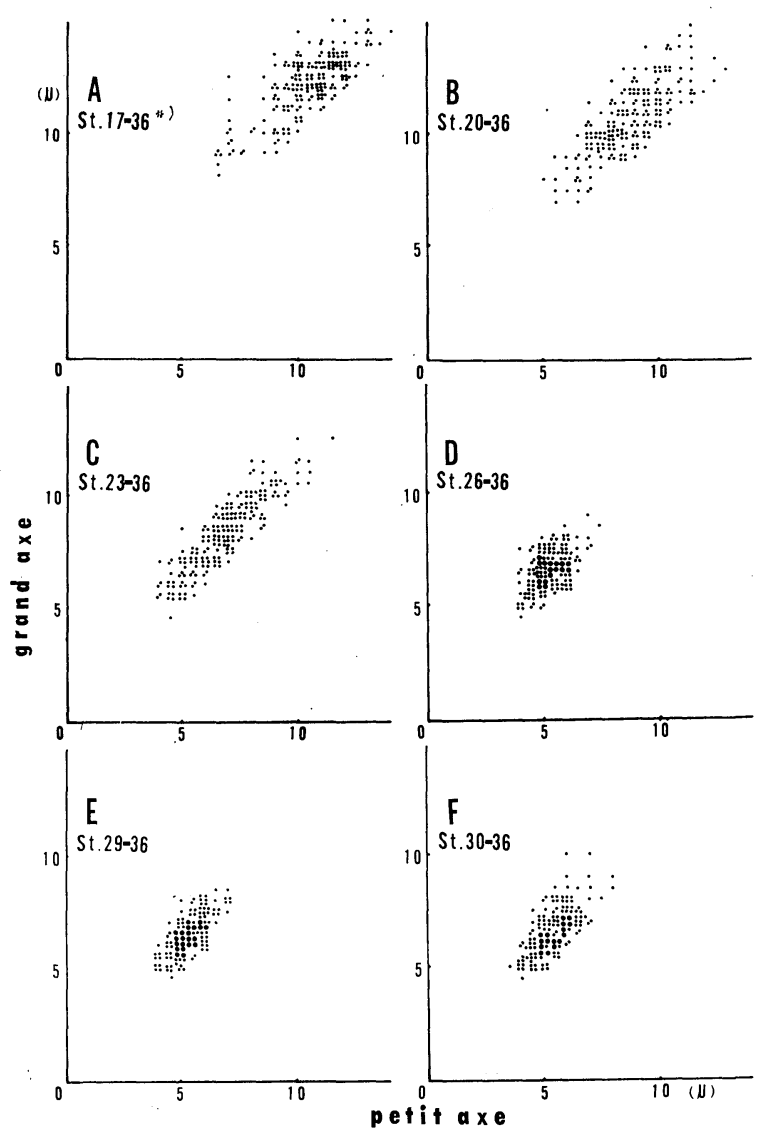

Fig. 2. Taille des moyaux cellulaires différenciés au stade 17 (A), 20 (B), 23 (C), 26 (D), 29 (E) et 30 (F). *) Les chiffres combinés indiquent que cet embryon a reçu une injection unique au stade 17 et qu'il a été fixé au stade 36 , et ainsi de suite.

des coupes sériées de $10 \mu$ d'épaisseur, qui ont été traitées ensuite pour l'autoradiographie en utilisant le procédé de Kopriwa et Leblond (1962)..$^{5)}$

Dans chaque cas, 200 noyaux cellulaires parmi les plus fortement marqués ont été photographiés au microscope à l'agrandissement de 400. Nous avons mesuré pour chacun d'eux le petit et le grand axe avec un micrométre (même agrandissement). Après avoir calculé d'après ces données originales l'aire de coupe des noyaux cellulaires $(S)$, en la considerant comme élliptique, nous avons groupé pour chaque cas les noyaux cellulaires le plus fortement marqués en classes de fréquence avec un intervalle de classe de $15 \mu^{2}$. Les résultats sont illustrés en histogramme à la figure 3 . Nous avons 


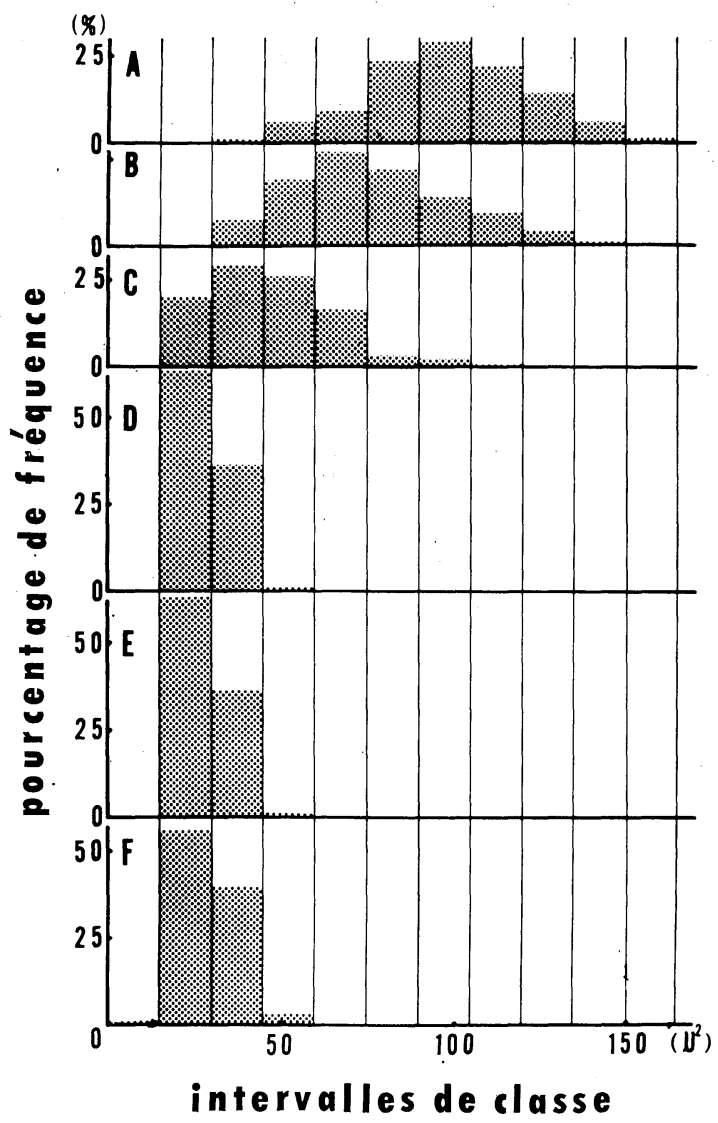

Fig. 3. Histogramme des aires de coupe des noyaux différenciés au stade 17 (A), 20 (B), 23 (C), 26 (D), 29 (E) et 30 (F).

ensuite calculé dans chaque cas la moyenne arithmétique des aires de coupe $(\bar{S})$, puis la déviation standard $(\sigma)$ selon la formule:

$$
\sigma=\sqrt{\frac{\sum(S-\bar{S})^{2}}{n-1}}
$$

dans laquelle: $n=$ nombre d'observations.

Les limites de confiance de la moyenne $(l)$ ont été calculées grâce à la formule:

$$
l=\bar{S} \pm \frac{\sigma}{\sqrt{n}} \cdot t
$$

dans laquelle: $\frac{\sigma}{\sqrt{n}}=$ erreur standard de la moyenne; $t=t$ de Student. La table de $t$ indique, pour un niveau $0.1 \%$ et pour un degré de liberté infini une valeur de $t=3.29$. On peut obtenir ainsi les limites de confiance des moyennes avec un coefficient de sécurité de $99.9 \%$. Enfin, pour mettre en évidence la différence de taille entre les noyaux 
Tableau

\begin{tabular}{ccccccccc}
\hline cas & $n$ & mode & $\bar{S}$ & $\sigma$ & $\frac{\sigma}{\sqrt{n}}$ & l.c. & $\bar{S}-\overline{S^{\prime}}$ & $\sigma_{D}$ \\
\hline A & 199 & 97.5 & 99.5 & 28.6 & 1.73 & $93.8-105.3$ & & \\
B & 196 & 67.5 & 76.5 & 23.0 & 1.65 & $71.0-81.9$ & 23.0 & 2.5 \\
C & 190 & 37.5 & 46.7 & 18.9 & 1.37 & $42.1-51.2$ & 29.8 & 2.2 \\
D & 199 & 22.5 & 28.2 & 7.7 & 0.54 & $26.3-30.0$ & 18.5 & 1.5 \\
E & 200 & 22.5 & 28.1 & 7.4 & 0.52 & $26.3-29.8$ & 0.1 & 0.8 \\
F & 200 & 22.5 & 29.5 & 8.2 & 0.60 & $27.5-31.6$ & 1.4 & 0.8 \\
\hline
\end{tabular}

Tableau des valeurs obtenues exprimées en $\mu^{2}$.

1. c. =Limites de confiance de $\bar{S}$ à $99.9 \%$.

cellulaires différenciés à des stades différents, nous avons calculé l'écart de la différence entre les moyennes $\left(3 \sigma_{D}\right)$ selon la formule suivante:

$$
\sigma_{D}=\sqrt{\frac{\sigma^{2}}{n}+\frac{\sigma^{\prime 2}}{n^{\prime}}} .
$$

Si la différence entre deux moyennes $\left(\bar{S}-\bar{S}^{\prime}\right)$ est supérieure au triple de l'écart de la différence entre les moyennes $\left(3 \sigma_{D}\right)$ on peut dire qu'il y a une différence hautement significative entre ces deux moyennes avec un coefficient de sécurité de $99.7 \%$.

Les données de mesure sur le petit et le grand axe des noyaux cellulaires le plus fortement marqués sont illustrées pour chaque cas en diagramme de dispersion à la figure 2, dans laquelle la fréquence est représentée par le nombre des points. Le simple examen de la figure 2 permet d'apercevoir aisément la tendance que les noyaux cellulaires différenciés avant le stade 26 (A, B, C et D) sont d'autant plus grands qu'ils se produisent plus tôt, tandis que ceux qui se différencient après le stade $26(\mathrm{D}, \mathrm{E}$ et $\mathrm{F})$ sont tous petits et presque de même taille. La figure 3 révèle que pour les cas $\mathrm{A}, \mathrm{B}, \mathrm{C}$ et $\mathrm{D}$ à mesure que le stade de différenciation s'avance la valeur la plus fréquente (mode) diminue progressivement, tandis que pour les cas $\mathrm{D}, \mathrm{E}$ et $\mathrm{F}$ la valeur modale demeure égale l'une à l'autre indépendamment de l'époque d'origine. Il en est de même des valeurs moyennes reportées dans le tableau.

L'intervalle de confiance permet d'évaluer si deux moyennes diffèrent significativement. Comme on le voit dans le tableau, les intervalles de confiance des cas A, B, C et D ne coïncident pas du tout, on a done un indice que ces cas diffèrent d'une façon hautement significative. Pour les cas D, E et F, cependant, les limites des intervalles de confiance coïncident entre 27.5 et 28.9 , il ne paraît 


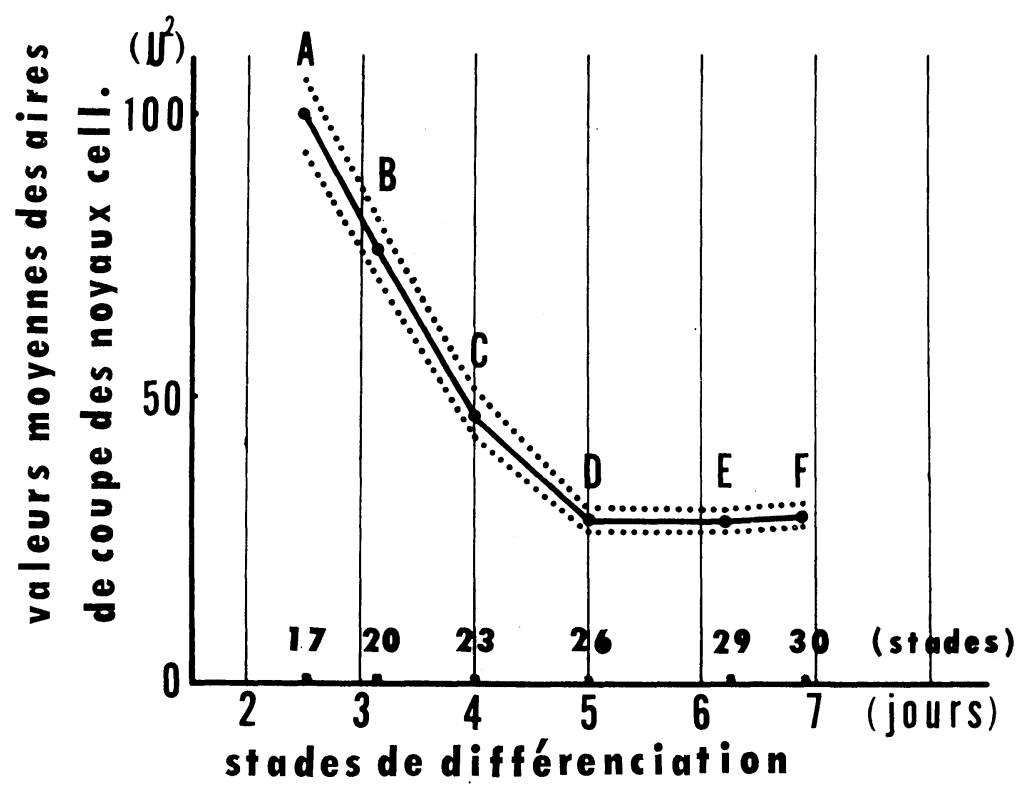

Fig. 4. Relation entre les moyennes des aires de coupe des noyaux cellulaires et leurs époques d'apparition. L'intervalle de confiance pour le niveau de confiance de $99.9 \%$ est limité par les deux courbes en pointillé.

donc pas y avoir différence significative entre ces trois derniers cas.

Dans le tableau ont été reportées aussi les valeurs de la différence entre les moyennes $\left(\bar{S}-\bar{S}^{\prime}\right)$ et les valeurs de l'écart de la différence entre les moyennes $\left(\sigma_{D}\right)$. Les résultats obtenus démontrent que la différence entre les moyennes est toujours supérieure au triple de l'écart de la différence entre les moyennes pour les cas A, B, C et D, tandis que pour les cas $\mathrm{D}, \mathrm{E}$ et $\mathrm{F}$, la différence entre les moyennes est inférieure au triple de l'écart de la différence entre les moyennes. Il en résulte qu'il y a une différence hautement significative entre les noyaux cellulaires différenciés après le stade 26 quant aux noyaux cellulaires différenciés après le stade 26 il n'y pas différence significative entre eux.

Avec les valeurs moyennes et avec les limites de l'intervalle de confiance nous avons tracé la courbe de la figure 4, qui montre directement la relation susmentionnée entre la taille des noyaux cellulaires et leur époque d'apparition.

Les névroglies dans la moelle épinière chez le poulet ne se différencient qu'au $7^{\text {me }}$ jour d'après Martin et Langman $(1965){ }^{6)}$ et qu'au $9^{\text {me }}$ jour d'incubation d'après Fujita (1964)..$^{7)}$ Les éléments que nous avons mis en question ici se sont tous différenciés avant le $7^{\text {me }}$ jour d'incubation, on pourrait donc les regarder presque tous pour des neurones. 
Bok $(1959)^{8)}$ a examiné la relation entre le volume nucléaire et celui de péricaryon des neurones dans l'écorce cérébrale chez le chat et conclu que le volume de péricaryon est exactement proportionnel au carré du volume nucléaire. Nous pourrions donc dire qu'au lieu des noyaux cellulaires les corps cellulaires sont d'autant plus grands qu'ils se différencient plus tôt.

D'après Herman et Lapham (1969) ${ }^{9)}$ les neurones de Betz, les cellules de Purkinje, les cellules pyramidales de la corne d'Ammon et de certains neurones de grande taille dans la moelle épinière chez le chat son tétraploïdes. Parce qu'il y a, dans l'écorce cérébrale, des neurones diploïdes de plus grande taille que les cellules de Purkinje et qu'il y a, dans la moelle épinière, des neurones octaploïdes de même taille que les neurones tétraploïdes, il ne semble pas qu'il y ait de corrélation linéaire entre la taille des neurones et la polyploïdie. Par contre, au moins au point de vue du développement nous avons montré qu'il y a une relation assez intime entre la taille des neurones et leur époque d'apparition.

Nous exprimons notre reconnaissance au Docteur Kenji Ikeda (Institute d'Electronique Médicale, Faculté de Médecine, Université de Tokyo) pour son assistance dans l'analyse statistique des données.

\section{Références}

1) Sidman, R. L. (1970): Autoradiographic methods and principles for study of the nervous system with thymidine- $\mathrm{H}^{3}$. 253-274, in Contemporary research methods in neuroanatomy, edited by W. J. H. Nauta and S. O. E. Ebbesson, Springer, Berlin.

2) Hinds, J. W. (1968): Autoradiographic study of histogenesis in the mouse olfactory bulb. I. Time of origine of neurons and neuroglia. J. Comp. Neur., 134, 287-304.

3) Kanemitsu, A. (1970): Cytoarchitecture de la moelle épinière chez le poulet envisagée au point de vue du développement, Etude autoradiographique. Proc. Japan Acad., 46, 77-82.

4) Hamburger, V., and H. L. Hamilton (1951): A series of normal stages in the development of the chick embryo. J. Morphol., 88, 49-92.

5) Kopriwa, B., and C. P. Leblond (1962): Improvements in the coating technique of radioautography. J. Histochem. Cytochem., 10, 269-284.

6) Martin, A., and J. Langman (1965): The development of the spinal cord examined by autoradiography. J. Embryol. exp. Morphol., 14, 25-35.

7) Fujita, S. (1964): Analysis of neuron differenciation in the central nervous system by tritiated thymidine autoradiography. J. Comp. Neur., 122, 311-328.

8) Bok, S. T. (1959): Histonomy of the cerebral cortex. Elsevier, Amsterdam.

9) Herman, C. J., and L. W. Lapham (1969): Neural polyploidy and nuclear volumes in the cat central nervous system. Brain Res., 15, 35-48. 